\title{
The use of imaging in COVID-19—results of a global survey by the International Society of Radiology
}

\author{
Ivana Blažić ${ }^{1}$ (D) • Boris Brkljačić ${ }^{2} \cdot$ Guy Frija $^{3}$
}

Received: 24 June 2020 / Revised: 12 August 2020 / Accepted: 31 August 2020 / Published online: 17 September 2020

(C) European Society of Radiology 2020

\begin{abstract}
Objectives This survey conducted by the International Society of Radiology and supported by the European Society of Radiology aimed to collect information regarding radiology departments' current practices in the management of patients with COVID-19.

Methods Responses from 50 radiology departments involved in the management of COVID-19 patients representing 33 countries across all continents were analyzed. The analysis revealed important variations in imaging practices related to COVID-19 across the world for different disease severity and various clinical scenarios.

Results Imaging is usually not performed in asymptomatic patients (69\% of institutions do not image) but is used at the end of confinement (in $60 \%$ of institutions). In the majority of institutions, chest imaging is used in suspected or confirmed patients with COVID-19 (89\% and 94\%). All imaging departments involved in this survey reported the use of imaging in COVID-19 patients showing severe symptoms or who were critically ill. However, there is a wide variation in imaging modality type used for each clinical scenario. The use of imaging is applied in line with existing guidelines and recommendations in $98 \%$ of institutions with structured reporting recorded in 58\% of institutions. The vast majority of institutions reported a significant impact of the COVID19 pandemic on the imaging department's routine activity $(83 \%)$.

Conclusion We believe that the results of this survey will help to understand current heterogeneities in radiology practice and to identify needs and gaps in the organization and function of radiology departments worldwide in relation to the COVID-19 pandemic. The results of this survey may inform the development of an overall strategy for radiology department organization and imaging protocols in pandemic conditions.

Key Points

- The results of this survey, which included responses from 50 radiology departments representing 33 countries, showed important variations in imaging practices related to COVID-19 across the world.

- While imaging is usually not performed in asymptomatic patients (69\% of institutions), it is used in suspected or confirmed patients with COVID-19, in COVID-19 patients showing severe symptoms or who were critically ill, and at the end of confinement (89\%, 94\%, 100\%, 100\%, 60\% of institutions, respectively). However, there is a wide variation in imaging modality type used for each clinical scenario.

- In 98\% of institutions, the use of imaging is applied in line with existing guidelines and recommendations, with structured reporting recorded in $58 \%$ of institutions. COVID-19 pandemic made a significant impact on the imaging department's routine activity in $83 \%$ of institutions.
\end{abstract}

Keywords COVID-19 pandemic $\cdot$ Radiology department $\cdot$ Survey

Electronic supplementary material The online version of this article (https://doi.org/10.1007/s00330-020-07252-3) contains supplementary material, which is available to authorized users.

Ivana Blažić

ivanablazic@yahoo.com

Radiology Department, Clinical Hospital Centre Zemun, Belgrade, Serbia
2 Department of Diagnostic and Interventional Radiology, University Hospital Dubrava, Zagreb, Croatia

3 University Paris-Centre, Paris, France 


\begin{tabular}{ll}
\multicolumn{2}{l}{ Abbreviations } \\
ACR & American College of Radiology \\
BSTI & British Society of Thoracic Imaging \\
CBR & Brazilian College of Radiology \\
COVID-19 & Coronavirus disease 2019 \\
CSR & Chinese Society of Radiology \\
CXR & Chest X-ray \\
ENT & Ears, nose, and throat \\
ESR & European Society of Radiology \\
ESTI & European Society of Thoracic Imaging \\
HAS & French National Health Authority \\
ICU & Intensive care units \\
ISR & International Society of Radiology \\
LUS & Lung ultrasound \\
PE & Pulmonary embolism \\
RANZCR & Royal Australian and New Zealand College \\
& of Radiologists \\
RCR & UK Royal College of Radiologists \\
RSR & Russian Society of Radiology \\
RT-PCR & Reverse transcriptase polymerase chain \\
& reaction \\
SERAM & Spanish Society of Radiology \\
SFR & French Society of Radiology \\
STR & Tunisian Society of Radiology \\
&
\end{tabular}

\section{Introduction}

A group of patients with atypical pneumonia was identified in Wuhan, China, in December 2019. The causative virus was named severe acute respiratory syndrome coronavirus-2 (SARS-CoV-2) and the associated disease - coronavirus disease 2019 (COVID-19) [1-3]. Soon thereafter, COVID-19 has spread rapidly throughout the world. The World Health Organization (WHO) declared the outbreak of public health emergency of international concern at the end of January 2020 and in March 2020 the global health situation was characterized as a pandemic outbreak.

Although the diagnosis of COVID-19 is confirmed by the identification of viral RNA in reverse transcriptase polymerase chain reaction (RT-PCR), chest imaging plays an important role in the diagnostic workup of patients with possible or suspected disease, in particular in settings where RT-PCR testing is not available or test results are delayed, as well as in patients showing respiratory symptoms associated with COVID-19 in whom RT-PCR tests are initially negative. Furthermore, chest imaging is used in the clinical management and follow-up of patients with COVID-19 in addition to laboratory parameters and clinical findings. Finally, in some patients with COVID-19, different imaging exams and image-guided procedures of various body sections have to be undertaken in line with proper indications [4].
In the context of the COVID-19 pandemic, all health facilities have been faced with an urgent need for setting up new organization models and adapting protocols regarding the management of patients with COVID-19. Radiology departments also had to adapt their workflow and to organize workforce according to the new circumstances. The International Society of Radiology (ISR) and the European Society of Radiology (ESR) recognized a need to get an insight of radiology department organization during the COVID-19 pandemic and therefore conducted this global survey with the aim to collect information regarding current practices of radiology departments in the management of COVID-19 patients.

\section{Material and methods}

The survey was conducted from the 6th to 26th of April 2020. For this purpose, an online questionnaire regarding the use of imaging in COVID-19 patients was prepared using Survey Monkey. The survey was sent to renowned representatives of ISR and ESR member countries.

The questionnaire was composed of six clinical scenarios regarding different levels of disease probability (suspected case/confirmed case), disease severity (asymptomatic/severe symptoms/critically ill patient), and patient management (hospital discharge). The use of imaging was assessed first in two clinical scenarios set up in situations of different probabilities of COVID-19-in symptomatic patients with suspected COVID-19 and in patients with confirmed disease, and secondly in three clinical scenarios with different disease severities - in asymptomatic subjects, in patients with severe symptoms and in critically ill patients. Furthermore, the use of imaging for decision-making related to the discharge of hospitalized patients with COVID-19 was investigated. Under each scenario, several questions were posed about the type of imaging modality performed (chest X-ray, chest CT, lung US, MRI) and the reason for imaging use in that particular clinical scenario. Additionally, three general questions on whether departments undertook the measures recommended by existing guidelines, structured reporting of imaging in patients with COVID-19, and the impact of the COVID-19 pandemic on radiological department's organization were included in the survey. Finally, at the end of the survey, respondents could add any type of comments in a free-text field. The survey questionnaire form is included in Annex 1.

\section{Results}

Information about the use of imaging in patients with suspected or confirmed COVID-19 was collected in order to assess current imaging practices in different clinical scenarios related to COVID-19. The survey gathered responses from 52 
institutions settled in 34 countries representing six continents as follows: 10 institutions from six countries in Africa, nine institutions from seven countries in Asia, one institution from Australia, 22 institutions from 15 countries in Europe, five institutions from two countries in North America, and five institutions from three countries in South America (Table 1).

Two radiology departments, one institution in Poland and one in The Netherlands, completed the survey, but since they have not been involved in the management of COVID-19 patients, data from those two institutions were excluded from further analysis. Finally, responses from 50 radiology departments from 33 countries were analyzed. The response rate was $88 \%$.

The questions in the survey referred to the use of imaging in patients with suspected or confirmed COVID-19 from asymptomatic to critically ill and at the end of confinement. For diagnostic workup and management purposes in patients with suspected or confirmed COVID-19, chest imaging was performed both on an outpatient basis and during the hospitalization, depending on the specific clinical scenario. Important variations in imaging practices related to COVID19 across the world have been revealed for different disease severity and various clinical scenarios.

In the majority of institutions (69\%), imaging is not performed in asymptomatic subjects. Of those who use imaging for the detection of lung disease related to COVID-19, 50\% use chest X-ray (CXR), 36\% chest CT, and 14\% both modalities (CXR and CT). The rationale for use of imaging is its ease of access $(57 \%)$ and because the results of imaging are faster than molecular test results $(36 \%)$, while in $7 \%$ of the institutions, molecular tests were not available.

In symptomatic patients with suspected COVID-19, the vast majority of imaging departments perform imaging (89\%), of which $37 \%$ use chest X-ray, 34\% chest CT and $29 \%$ both modalities (CXR and CT). Faster results than molecular tests (51\%) and easy access (39\%) were the main reasons for imaging use, while in $5 \%$ of the institutions there was no access to molecular tests. Geographical variations in the use of imaging in symptomatic patients with suspected COVID-19 are presented in Fig. 1.

In patients with confirmed COVID-19, imaging is performed in the vast majority of imaging departments (94\%), of which $22 \%$ use chest X-ray, $28 \%$ chest CT, and $43 \%$ both $\mathrm{CXR}$ and $\mathrm{CT}$. In some institutions, the combination of three modalities (CXR, CT and lung ultrasound [LUS] or CT, LUS and MRI [ $4 \%$ and 2\%]) is used. The purpose of imaging use in patients with confirmed COVID-19 was the detection of lung disease $(96 \%)$ or thrombotic complication $(52 \%)$ related to COVID-19. Geographical variations in the use of imaging in patients with confirmed COVID-19 are shown in Fig. 2.

All imaging departments analyzed in this survey reported the use of imaging in patients with COVID-19 showing severe symptoms or who were critically ill. For those reasons, chest
Table 1 List of countries and number of institutions from each country that participated in the survey

\begin{tabular}{|c|c|c|}
\hline & Country & Number of institutions \\
\hline 1. & Algeria & 1 \\
\hline 2. & Argentina & 1 \\
\hline 3. & Australia & 1 \\
\hline 4. & Austria & 1 \\
\hline 5. & Belgium & 1 \\
\hline 6. & Brazil & 3 \\
\hline 7. & Canada & 1 \\
\hline 8. & Chile & 1 \\
\hline 9. & China & 2 \\
\hline 10. & Croatia & 1 \\
\hline 11. & Egypt & 1 \\
\hline 12. & France & 4 \\
\hline 13. & Germany & 1 \\
\hline 14. & Greece & 1 \\
\hline 15. & Hungary & 1 \\
\hline 16. & India & 1 \\
\hline 17. & Iran & 1 \\
\hline 18. & Ireland & 1 \\
\hline 19. & Israel & 1 \\
\hline 20. & Italy & 3 \\
\hline 21. & Japan & 1 \\
\hline 22. & Kenya & 2 \\
\hline 23. & Netherlands & 2 \\
\hline 24. & Poland & 1 \\
\hline 25. & Russian Federation & 1 \\
\hline 26. & South Africa & 4 \\
\hline 27. & South Korea & 1 \\
\hline 28. & Spain & 2 \\
\hline 29. & Taiwan & 2 \\
\hline 30. & Tunisia & 1 \\
\hline 31. & Turkey & 1 \\
\hline 32. & Uganda & 1 \\
\hline 33. & UK & 1 \\
\hline \multirow[t]{2}{*}{34.} & USA & 4 \\
\hline & & 52 \\
\hline
\end{tabular}

X-ray is performed in $10 \%$ and $33 \%$ of institutions respectively, chest CT in $31 \%$ and $19 \%$ of institutions, both CXR and $\mathrm{CT}$ in $49 \%$ and $23 \%$ of institutions, and three imaging modalities (CXR, CT, and LUS) in $6 \%$ and $13 \%$ of institutions. Additionally, in patients with severe symptoms, the combination of chest CT and LUS was used in $4 \%$ of institutions, while in $13 \%$ of institutions the combination of CXR and LUS was used in patients who were critically ill. The purpose of imaging use in patients showing severe symptoms or being critically ill was the detection of lung disease (98\% and $88 \%$ respectively) or thrombotic complication (73\% and $54 \%$ 


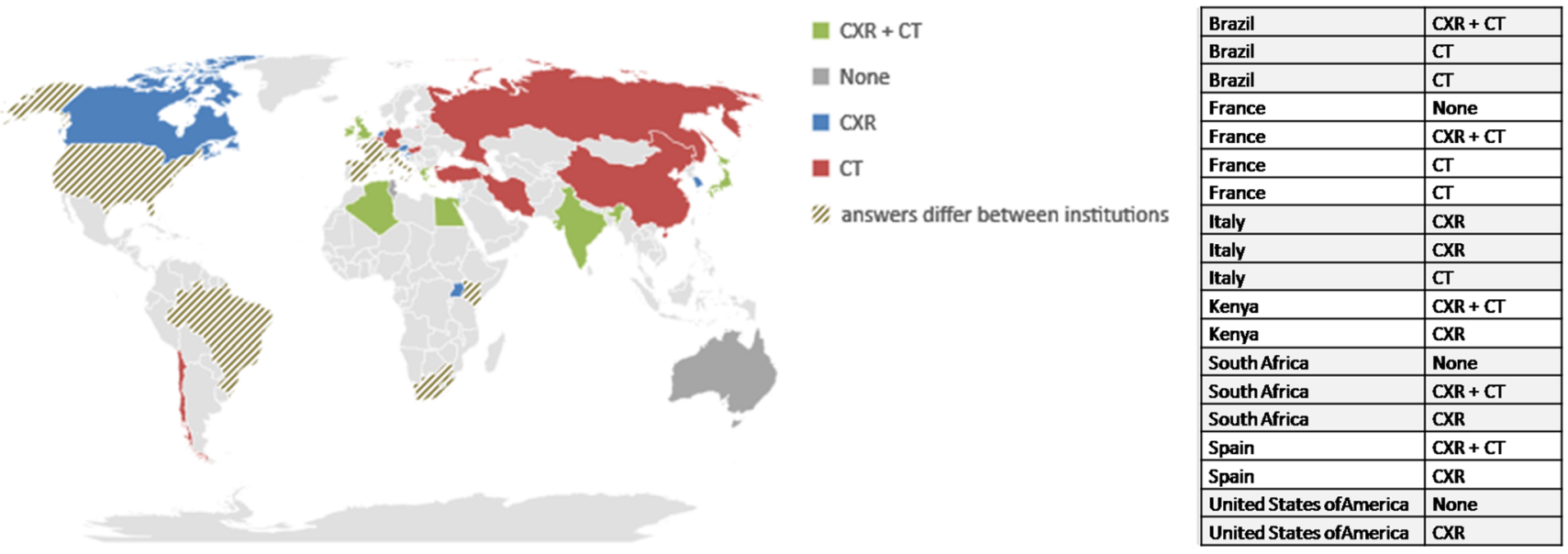

Fig. 1 Map chart showing geographical variations in the use of imaging in patients with suspected COVID-19 (different answers from institutions in the same country are given in the side table) in the use of imaging in patients with COVID-19 showing severe symptoms or who were critically ill are shown in Figs. 3 and 4.

At the end of confinement, imaging is used in $60 \%$ of institutions (45\% CXR, 38\% CT, and $17 \%$ both modalities) in order to follow-up lung disease. Graphical representation of the general use of imaging related to COVID-19 and the use of different imaging techniques in various clinical scenarios are given in Figs. 5 and 6.

The use of imaging is organized in line with guidelines and recommendations in $98 \%$ of institutions $(70 \%$ of those institutions follow national guidelines or recommendations, 55\% local guidelines or recommendations, and $28 \%$ other guidance, e.g., from international professional societies). Structured reporting is performed in 58\% of institutions analyzed in this survey. In all institutions, reports were made by radiologists. The vast majority of institutions reported a significant impact of the COVID-19 pandemic on the imaging respectively) related to COVID-19. Geographical variations

department's routine activity (83\%), $8 \%$ reported moderate, and $8 \%$ low impact.

\section{Discussion}

SARS-CoV 2, a new coronavirus infection, is responsible for the respiratory disease COVID-19 for which the diagnosis relies on the identification of viral RNA by the reversetranscription polymerase chain reaction [1-3]. However, imaging can also identify and characterize pulmonary involvement of COVID-19 [4].

One can see from the survey that the practice of imaging in COVID-19 differs throughout the world, and environments differ considerably, especially regarding the utilization of conventional chest $\mathrm{X}$-ray. To summarize broadly what was reported in the survey, conventional chest X-ray is mostly used in intensive care units (ICU) at patient bedside and for the follow-up of patients during treatment. Mobile X-ray units

\begin{tabular}{|c|c|c|}
\hline & & \\
\hline & China & $\mathrm{CXR}+\mathrm{CT}$ \\
\hline \multirow{2}{*}{$\begin{array}{l}=\mathrm{CXR}+\mathrm{CT} \\
=\mathrm{CT}\end{array}$} & China & CT \\
\hline & France & CT + US + MRI \\
\hline $\mathscr{M}$ answers differ between institutions & France & $C T$ \\
\hline CXR & France & CT \\
\hline \multirow{12}{*}{ None } & France & $\mathrm{CT}$ \\
\hline & Italy & $\mathrm{CXR}+\mathrm{CT}$ \\
\hline & Italy & $\mathrm{CXR}+\mathrm{CT}$ \\
\hline & Italy & $\mathrm{CXR}+\mathrm{CT}+\mathrm{US}$ \\
\hline & Kenya & $\mathrm{CXR}+\mathrm{CT}$ \\
\hline & Kenya & CXR \\
\hline & South Africa & $\mathrm{CXR}+\mathrm{CT}$ \\
\hline & South Africa & $\mathrm{CXR}+\mathrm{CT}$ \\
\hline & South Africa & CXR \\
\hline & South Africa & CXR \\
\hline & Spain & $C X R+C T+U S$ \\
\hline & Spain & None \\
\hline
\end{tabular}

Fig. 2 Map chart showing geographical variations in the use of imaging in patients with confirmed COVID-19 (different answers from institutions in the same country are given in the side table) 


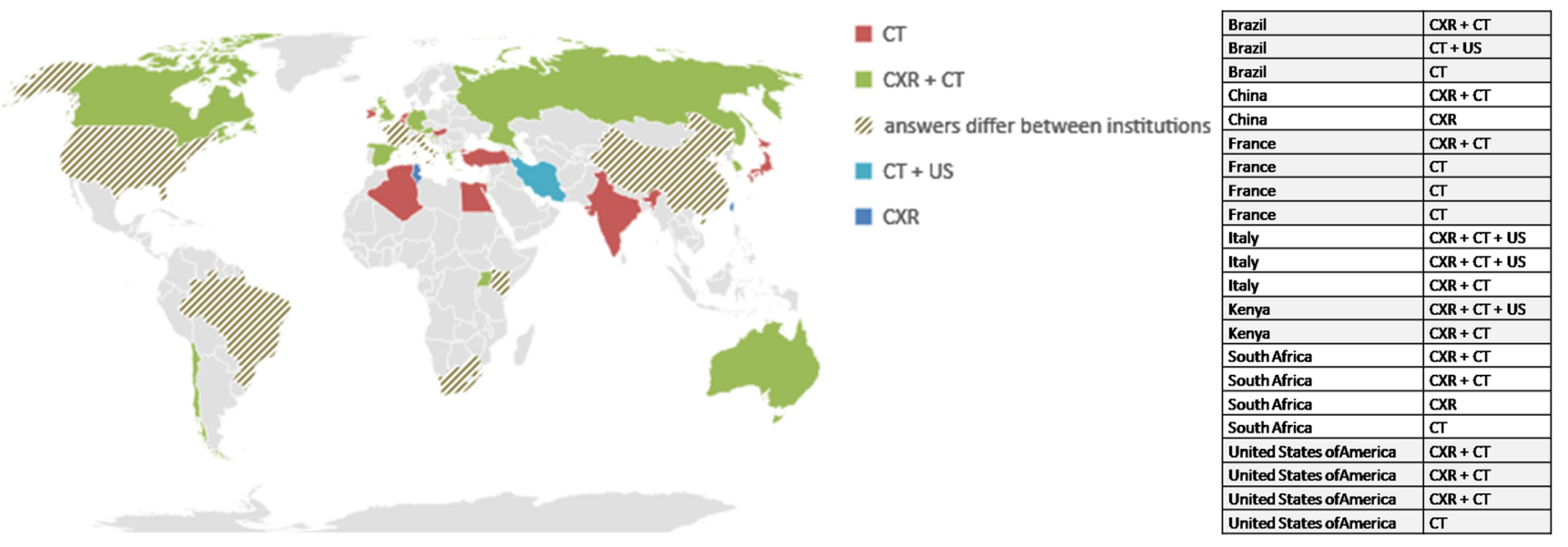

Fig. 3 Map chart showing geographical variations in the use of imaging in COVID-19 patients showing severe symptoms (different answers from institutions in the same country are given in the side table)

have a huge role in the COVID-19 pandemic. Ultrasound is used for bedside imaging and in the ICU, mostly by intensivists, usually using small mobile US units for pointof-care ultrasound. The use of CT varies considerably; routine screening CT for the identification of COVID-19 pneumonia is not recommended by most radiology societies, but CT may be performed in patients who are under investigation. Most agree to use contrast-enhanced chest $\mathrm{CT}$ to rule out pulmonary embolism (PE) and for the evaluation of lung volume under different pressures and changes in ventilation [5-17]. It is known that CXR is not as sensitive as CT [5] for the detection of ground-glass opacities, which are the main imaging features of COVID-19 pneumonia, but CXR is in general much easily accessible than CT.

Survey results demonstrate that the most applied imaging modalities are CXR, using mobile X-ray units for hospitalized patients, and chest CT. In general, X-ray is used more frequently than $\mathrm{CT}$ in asymptomatic patients, which probably reflects easier access to X-ray equipment. CXR is mostly used for the ICU at patient bedside and for the follow-up of patients during treatment. In critically ill patients, bedside CXR with portable X-ray units has advantages over CT because it is difficult and cumbersome to transport critically ill patients to CT units. LUS is in practice mostly used by intensive care physicians using point-of-care ultrasound at patient bedside. The utilization of CT depends a lot on local circumstances in a specific area since inequality in health care access is huge around the world. European and Chinese professional societies advocate for the use of CT more than others [5, 6], but most societies worldwide do not recommend routine use of CT for the identification of COVID-19 pneumonia, although CT may be performed in patients who are under investigation for possible complications [7-17]. Most agree to use contrastenhanced chest $\mathrm{CT}$ (CT angiography) to rule out $\mathrm{PE}$ and other complications related to ventilation. The fact that $\mathrm{CT}$ is used more frequently in symptomatic patients and to detect complications reflects advantages that $\mathrm{CT}$ as a modality offers compared with CXR. Patients with mild symptoms are very common, and one could conclude that imaging is not indicated in patients with negative COVID-19 tests, while it is

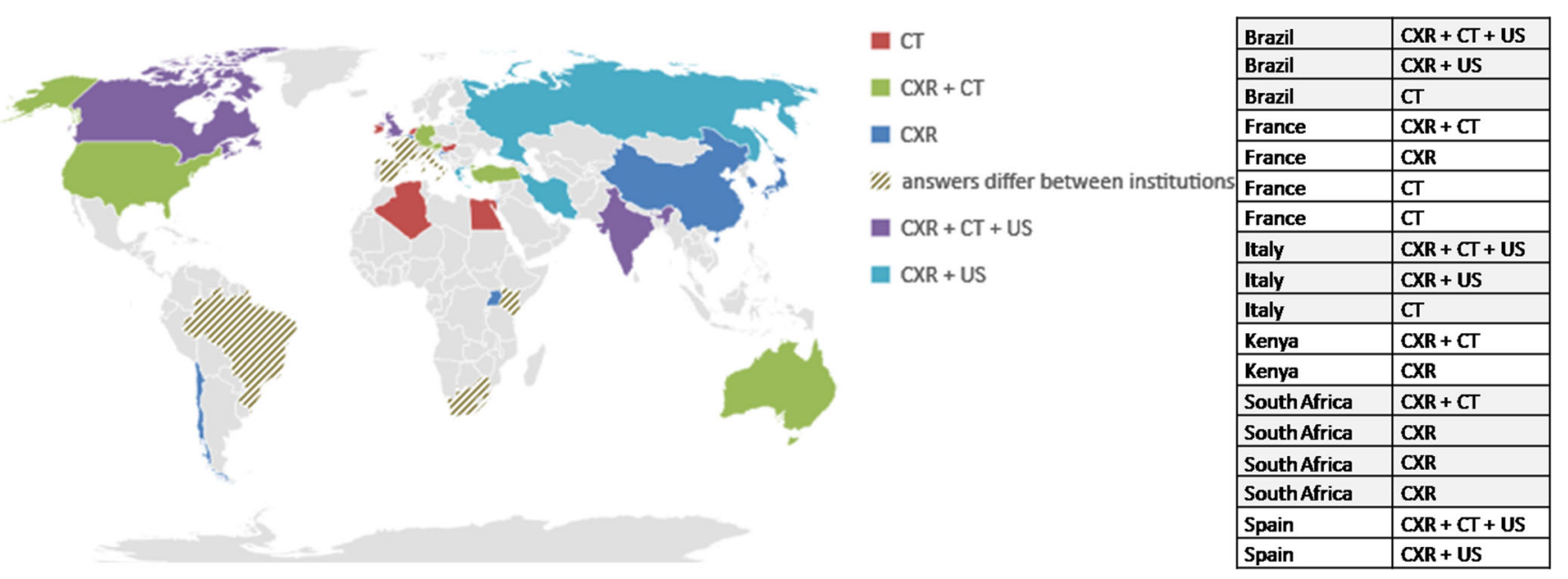

Fig. 4 Map chart showing geographical variations in the use of imaging in critically ill patients with COVID-19 (different answers from institutions in the same country are given in the side table) 
a

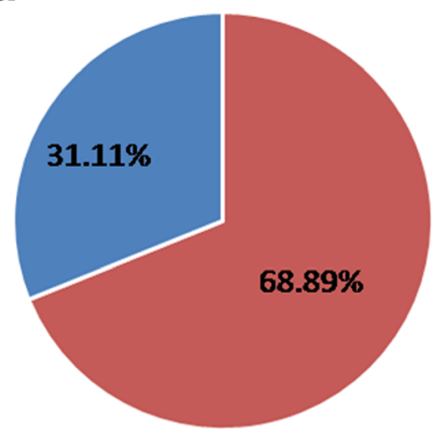

d

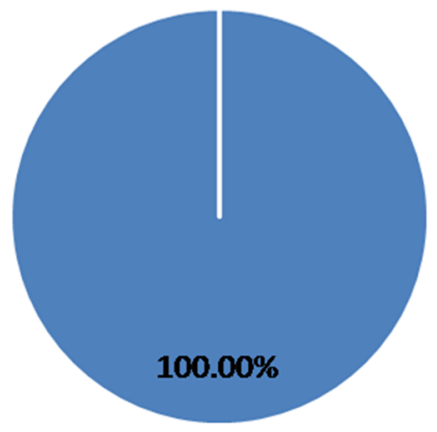

b

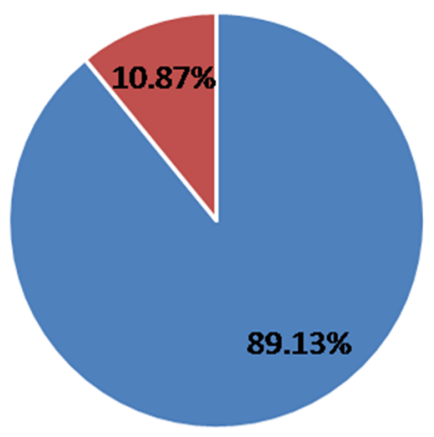

e

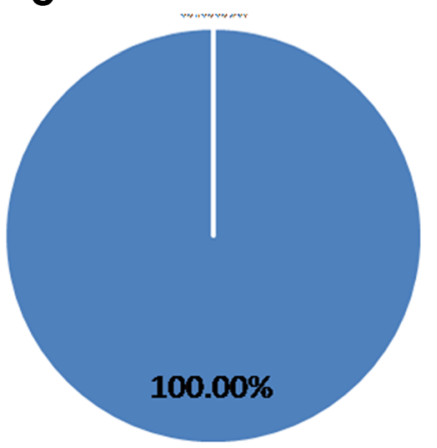

C

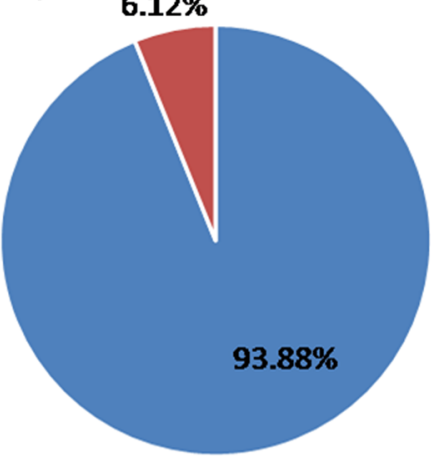

$\mathbf{f}$

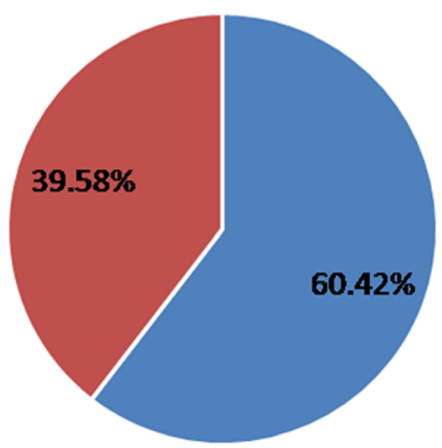

imaging no imaging

Fig. 5 Use of imaging related to COVID-19: in asymptomatic patients (a), symptomatic patients suspected of COVID-19 (b), confirmed patients (c), patients with severe symptoms (d), critically ill patients (e), and at the end of confinement (f)

indicated only in patients with mild features consistent with COVID-19, any pre-test probability, and unavailable or positive COVID-19 test if there are risk factors for disease progression. Also, the discrepancies are present between the guidelines regarding the performance of CT periodically on a routine basis. Although imaging is not used routinely in diagnosing COVID-19, in many cases patients have incidentally detected findings on conventional CXR or chest CT that might point to COVID-19 pneumonia as a possible cause of findings.

Our survey showed that imaging is used in $60 \%$ of institutions at the end of confinement. The WHO rapid advice guide on the use of chest imaging in COVID-19 does not recommend using chest imaging for hospitalized patients with COVID-19 whose symptoms are resolved to inform the decision regarding discharge. However, imaging in those circumstances may be useful in patients who have had a severe form of COVID-19 or pre-existing chronic lung disease [18].

The guidelines on the use of imaging in COVID-19 differ at a smaller or larger extent depending on the country or region and the level of and access to healthcare in the respective country or area of the world and it seems that they also reflect the local situation of the COVID-19 pandemic. In this section, we present recommendations of professional societies from countries with the largest proportions of COVID-19 patients, keeping at least one representative from each continent.

According to the Chinese Society of Radiology (CSR), CXR is not recommended for use on admission due to low sensitivity, and the first choice for radiological examination of COVID-19 is CT scan. CSR recommends repeating chest CT within 3-5 days for newly diagnosed patients with initially negative chest CT and within 5-7 days for patients with atypical clinical manifestations of diagnosed disease. For severe patients, bedside CXR can be used to observe the progression of the disease [6].

The Fleischner Society, an international multidisciplinary medical society dedicated to thoracic radiology, does not recommend imaging as a screening test for COVID-19 in asymptomatic individuals, while for symptomatic patients, the detailed recommendations are stratified based on the severity of symptoms (mild vs. moderate to severe), pre-test probability (any vs. low vs. high), and COVID-19 testing [7]. In patients with mild features consistent with COVID-19, any pre-test probability, and negative COVID-19 test, imaging is not 


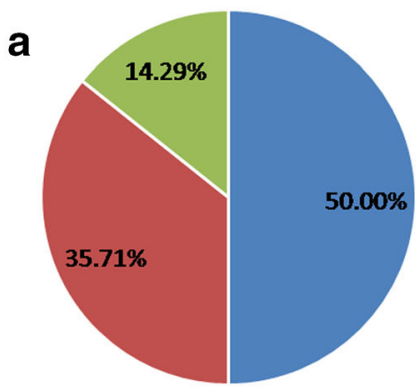

b
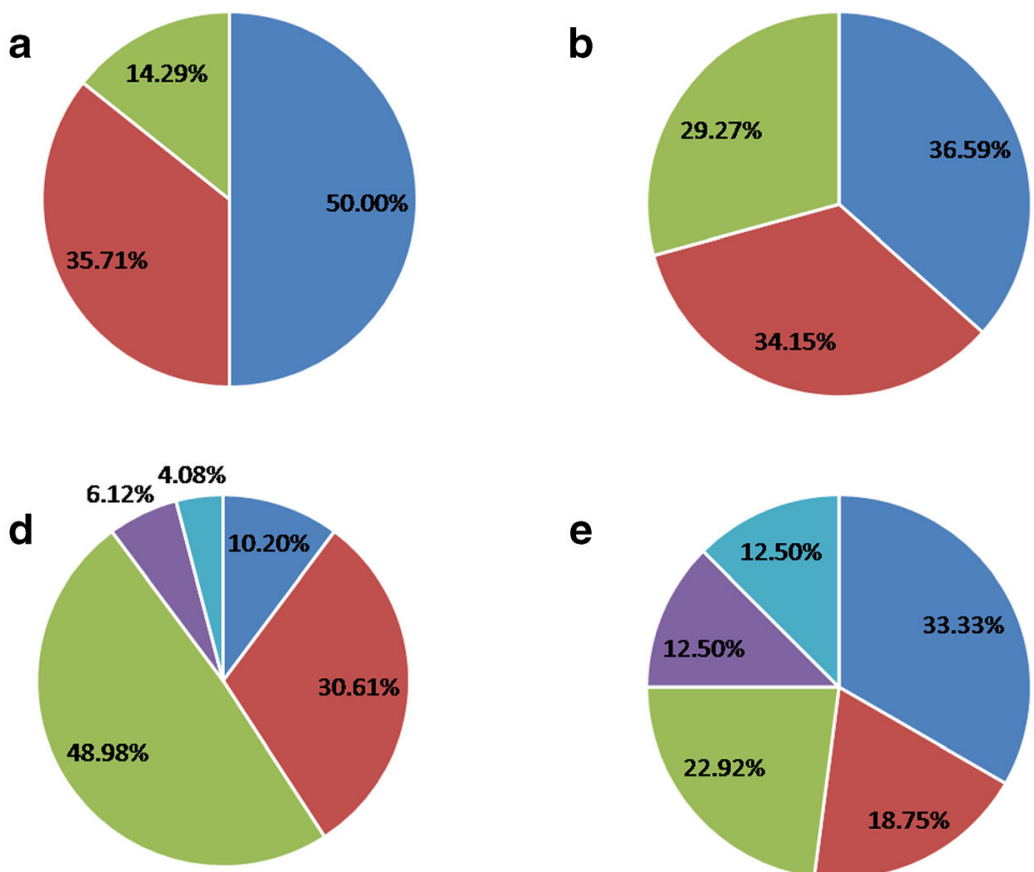
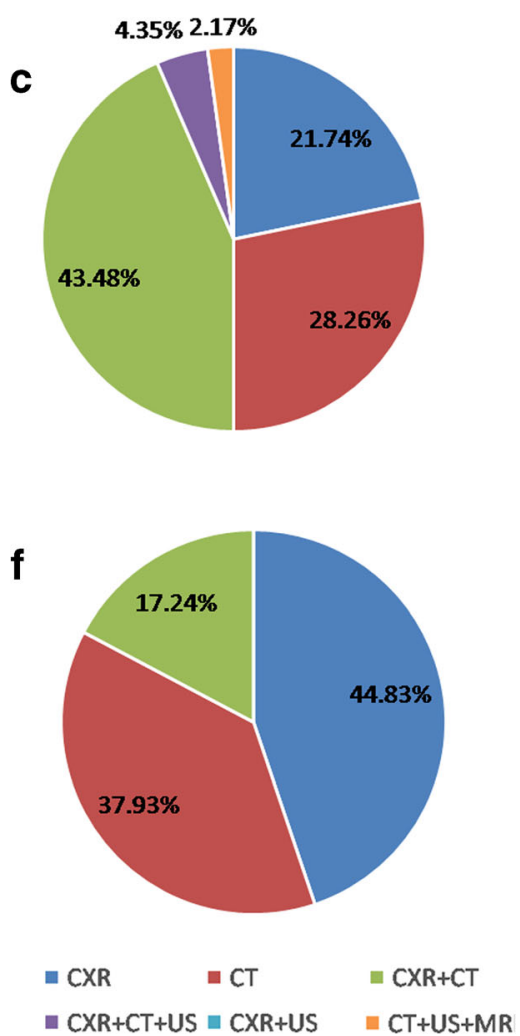

Fig. 6 Imaging technique used related to COVID-19 (if imaging is performed): in asymptomatic patients (a), symptomatic patients suspected of COVID-19 (b), confirmed patients (c), patients with severe symptoms (d), critically ill patients (e), and at the end of confinement (f)

indicated. Imaging is indicated in patients with mild features consistent with COVID-19, any pre-test probability, and unavailable or positive COVID-19 test if there are risk factors for disease progression; in patients with moderate to severe features consistent with COVID-19 and high pre-test probability regardless of COVID-19 test results; and in patients with COVID-19 showing worsening respiratory status.

The European Society of Radiology (ESR) and European Society of Thoracic Imaging (ESTI) recommend that CT should not be performed as a screening test in patients with mild or no symptoms [5]. In patients with suspected or confirmed COVID-19, chest X-ray is not recommended as the first-line technique since it is not sensitive for the detection of ground-glass opacities characteristic of COVID-19 pneumonia and should be restricted to the follow-up of patients admitted to ICU who are too fragile to be sent for CT. In those patients, ultrasound at the bedside is also recommended for the detection of complications such as pleural effusions or a pneumothorax under mechanical ventilation. If supplementary oxygen is needed in patients with limited disease extension, other diagnoses, especially PE, should be suspected and an additional contrast-enhanced CT may be indicated. Repeat $\mathrm{CT}$ scans are indicated in cases with suspected complications (e.g., PE, superinfection), but indications for repeat $\mathrm{CT}$ scans should be considered carefully, as the transport of patients from the ward to the CT unit is associated with the risk of contamination of personnel and other patients. In patients who are recovering, a repeat $\mathrm{CT}$ is not necessary.

The French Society of Radiology (SFR) advises against the use of chest CT for screening in patients without signs of severity and without comorbidities. Non-contrast high-resolution chest CT is indicated in patients under hospital care with suspected or confirmed COVID-19 diagnosis and initial or secondary signs of clinical severity (e.g., dyspnea, desaturation) [8]. In COVID-19-positive intensive care patients who are worsening, contrast-enhanced CT should be performed. The French National Health Authority (HAS) does not recommend chest imaging for screening purposes on patients without signs of severity for the diagnosis of COVID-19 either [9]. However, they advise utilization of chest CT in the absence of rapid biological tests for the detection of silent lung lesions in adult patients with unknown COVID-19 status who need to undergo emergency interventions (ENT, oncology, stroke, hemorrhage, etc.). Chest CT is indicated to guide the management and monitoring of pulmonary symptoms in patients with COVID-19.

The British Society of Thoracic Imaging (BSTI) is far more restrictive regarding the use of chest $\mathrm{CT}$. They do not see the practical value of using CT for asymptomatic patients regardless of the level of clinical suspicion and for patients with high 
clinical suspicion but negative initial RT-PCR [10]. According to BSTI, chest CT should be performed as an initial diagnostic tool, if a chest radiograph was normal or was not/ could not be performed and there is a lack of initial RT-PCR testing availability. In patients with COVID-19 RT-PCRpositive results, BSTI does not envisage a role for CT. Similarly, the UK Royal College of Radiologists (RCR) advises that there is no role for $\mathrm{CT}$ in the diagnostic assessment for patients with suspected COVID-19 [11].

The Spanish Society of Radiology (SERAM) recommends the use of imaging based on clinical and laboratory data [12]. Imaging is not indicated for COVID-19 screening in asymptomatic patients, but is indicated if patients have acute respiratory illness including pneumonia, even without fever or acute deterioration of chronic cardiorespiratory disease, or in patients with acute respiratory symptoms who are immunosuppressed. Also, chest $\mathrm{CT}$ is recommended in patients with an urgent indication of any surgery who cannot wait for the PCR result, regardless of the presence of COVID-19 symptoms. Imaging techniques can be considered a diagnostic alternative in the management of emergency patients, especially in cases where access to RT-PCR tests is limited or there is a clinical-radiological suspicion of a false-negative finding of the PCR. Chest CT is advised in patients with high clinical suspicion of COVID-19 at admission, negative PCR, and inconclusive or suspicious CXR for COVID-19. In hospitalized patients, imaging should not be obligatory, but must be based on clinical evolution. Patients with confirmed COVID-19 and clinical worsening with suspected PE or bacterial superinfection and the appearance of pleural effusion require chest $\mathrm{CT}$.

The Russian Society of Radiology (RSR) advises against $\mathrm{X}$-ray and CT in asymptomatic patients [13]. CXR is recommended in the presence of symptoms and clinical signs of acute respiratory viral infections, and mobile radiography is considered an important tool for diagnosis of lung pathology in ICUs. Chest CT is recommended for the initial evaluation of the lungs of patients with severe and progressive forms of the disease. Lung ultrasound is considered an additional imaging method that does not replace or exclude X-ray and CT. Imaging is also used for the follow-up of the disease, to assess whether a patient should be transferred to the intensive care unit, and for patient discharge.

According to the American College of Radiology (ACR) recommendations, $\mathrm{CT}$ should not be used as a screening test or as a first-line test for diagnosis in patients with suspected COVID-19, but chest CT should be sparingly used and reserved for hospitalized and symptomatic patients with specific clinical indications for CT [14]. For patients known to have tested positive or persons under investigation for COVID-19, the ACR recommends that practitioners minimize the use of MRI except where absolutely necessary. In some cases, the use of alternative imaging methods such as point-of-care ultrasound or portable X-ray equipment may be appropriate.
The Brazilian College of Radiology (CBR) does not recommend imaging in asymptomatic patients with suspected COVID-19 infection [15]. In PCR/Anti-IgM-positive patients with mild to moderate symptoms, any imaging exam is recommended. In patients with suspected COVID-19 infection with severe symptoms who are hospitalized, chest CT is advised if complications are suspected (e.g., PE, overlapping bacterial infection).

The Royal Australian and New Zealand College of Radiologists (RANZCR) does not recommend the use of chest CT for management of COVID-19 due to poor sensitivity and radiation exposure risk and strongly advises against conducting routine chest $\mathrm{CT}$ scans for individuals undergoing emergency surgery in Australia and New Zealand [16].

The Tunisian Society of Radiology (STR) does not recommend the use of CXR or CT in patients with suspicious but not confirmed COVID-19 without clinical signs of severe disease, comorbidities, or who are not hospitalized [17]. CXR is indicated in patients who require hospitalization. Indications for $\mathrm{CT}$ are discussed for hospitalized patients on a case-to-case basis depending on the possible complications.

Our study has some limitations. We have not applied any strict inclusion criteria for the survey. Instead, it was sent to renowned representatives of ISR and ESR member countries which represents a selection bias. Questions on the resources available for diagnostic workup and management of patients with suspected or confirmed COVID-19 patients in health centers have not been included in the survey. The impact of the imaging findings on clinical decision-making and change in the clinical management was not investigated either. It is planned to cover those issues by a follow-up survey.

Guidelines and recommendations of national and regional professional societies differ to a certain degree. The results of our survey are mostly in accordance with the guidelines of the relevant authority in the respective country or region. As a conclusion, it is obvious that the practice of imaging in COVID-19 differs throughout the world, especially regarding the utilization of conventional chest X-ray and computed tomography; we believe that the results of this survey will help to understand current practice heterogeneities and to identify needs and gaps in the organization and function of radiology departments worldwide in relation to the COVID-19 pandemic. The results of this survey may inform the development of an overall strategy for radiology department organization and imaging protocols in pandemic conditions.

Acknowledgments The International Society of Radiology and the European Society of Radiology gratefully acknowledge the contribution by all the institutions which took part in this survey.

We would also like to thank the staff of the European Society of Radiology (ESR), Martina Szucsich and Monika Hierath, for their contribution in communication with the institutions and for assembling data.

Funding The authors state that this work has not received any funding. 


\section{Compliance with ethical standards}

Guarantor The scientific guarantor of this publication is Professor Guy Frija.

Conflict of interest The authors of this manuscript declare no relationships with any companies, whose products or services may be related to the subject matter of the article.

Statistics and biometry No complex statistical methods were necessary for this paper.

Informed consent Written informed consent was not required for this study because it was a survey and all institutions' representatives agreed to participate in the survey.

Ethical approval Institutional Review Board approval was not required because it was a survey and all institutions representatives agreed to participate in the survey.

\section{Methodology}

- Observational multicenter study based on a survey

\section{References}

1. Zhou P, Yang XL, Wang XG et al (2020) A pneumonia outbreak associated with a new coronavirus of probable bat origin. Nature 579(7798):270-273

2. Zhu N, Zhang D, Wang W et al (2020) A novel coronavirus from patients with pneumonia in China, 2019. N Engl J Med 382(8): 727-733

3. Lu R, Zhao X, Li J et al (2020) Genomic characterisation and epidemiology of 2019 novel coronavirus: implications for virus origins and receptor binding. Lancet 395(10224):565-574

4. Manna S, Wruble J, Maron SZ et al (2020) COVID-19: a multimodality review of radiologic techniques, clinical utility, and imaging features. Radiology Cardiothoracic Imaging 2:3. https:// doi.org/10.1148/ryct.2020200210

5. Revel M-P, Parkar AP, Prosch H et al (2020) COVID-19 patients and the radiology department - advice from the European Society of Radiology (ESR) and European Society of Thoracic Imaging (ESTI). Eur Radiol. https://doi.org/10.1007/s00330-020-06865-y

6. Chinese Society of Radiology (2020) Radiological diagnosis of new coronavirus infected pneumonitis: expert recommendation from the Chinese Society of Radiology (First Edition). Chin J Radiol 54(00):E001-E

7. Rubin GD, Ryerson CJ, Haramati LB et al (2020) The role of chest imaging in patient management during the COVID-19 pandemic: a multinational consensus statement from the Fleischner Society. Chest 158(1):106-116

8. Société Française de Radiologie (2020) Les recommandations de la Société Française de Radiologie dans un contexte de COVID-19.
Available via https://ebulletin.radiologie.fr/covid19. Accessed 15 Jun 2020

9. Haute Autorité de Santé (2020) Réponses rapides dans le cadre du COVID-19 - indications du scanner thoracique. Available via https://ebulletin.radiologie.fr/actualites-covid-19/compte-rendutdm-thoracique-iv Accessed 15 Jun 2020

10. Nair A, Rodrigues JCL, Hare S et al (2020) A British Society of Thoracic Imaging statement: considerations in designing local imaging diagnostic algorithms for the COVID 19 pandemic. ClinRadiol 75(5):329-334

11. The Royal College of Radiologists (2020) The role of CT in patients suspected with COVID-19 infection. Available via https:// www.rcr.ac.uk/college/coronavirus-covid-19-what-rcr-doing/ clinical-information/role-ct-chest/role-ct-patients. Accessed 15 Jun 2020

12. Sociedad Española de Radiología Médica (2020) La radiología des de la aparición de la infección COVID-19. Análisis y recommendaciones Available via www.serames/index.php/seramrss/1463-nuevo-documento-la-radiologia-desde-la-aparacion-dela-infeccion-covid19 Accessed 15 Jun 2020

13. Sinitsyn VE, Tyurin IE, Mitkov VV (2020) Consensus guidelines of Russian Society of Radiology (RSR) and Russian Association of Specialists in Ultrasound Diagnostics in Medicine (RASUDM) role of imaging (X-ray, CT and US) in diagnosis of COVID-19 pneumonia (version 2). J Radiol Nucl Med 101(2):72-89 (in Russian)

14. American College of Radiology (2020) ACR recommendations for the use of chest radiography and computed tomography (CT) for suspected COVID-19 infection. Available via https:/www.acr.org/ Advocacy-and-Economics/ACR-Position-Statements/ Recommendations-for-Chest-Radiography-and-CT-for-SuspectedCOVID19-Infection. Accessed 15 Jun 2020

15. Colégio Brasileiro de Radiologia e Diagnóstico por Imagem (2020) Recomendações de uso de métodos de imagem para pacientes suspeitos de infecção pelo COVID-19. Available via https://cbr. org.br/recomendacoes-de-uso-de-metodos-de-imagem-parapacientes-suspeitos-de-infeccao-pelo-covid-19. Accessed 15 Jun 2020

16. Royal Australia and New Zealand College of Radiologists (2020) Guidelines for CT chest and chest radiograph reporting in patients with suspected COVID-19 infection. Available via www.ranzcr. com/college/document-library/guidelines-for-ct-chest-and-chestradiograph-reporting-in-patients-with-suspected-covid-19infection. Accessed 15 Jun 2020

17. Tunisian Society of Radiology (2020). Épidémie de Covid-19 Recommandations pour l'imagerie (Société Tunisienne de Radiologie et Collège d'Imagerie Médicale, Biophysique et médecine nucléaire). Available via http://strtn.org/media/articles/ Proc\%C3\%A9dures\%20\%20pour\%20l'imagerie\%20covid\%20\% 20STR\%20INEAS.pdf. Accessed 15 Jun 2020

18. World Health Organization (2020) Use of chest imaging in COVID-19: a rapid advice guide. World Health Organization, Geneva. Available via https://www.who.int/publications/i/item/ use-of-chest-imaging-in-covid-19. Accessed 27 Jul 2020

Publisher's note Springer Nature remains neutral with regard to jurisdictional claims in published maps and institutional affiliations. 